\title{
The unethical use of BMI in contemporary general practice
}

We seem to be regularly force-fed the idea that the body-mass index (BMI) is an appropriate method by which to measure obesity in our patients. Indeed, Read Code $22 \mathrm{~K} 5$ implies that obesity is only attained when the BMI is calculated as 30 or greater. However this is not only overly simplistic, but such a glib understanding can in fact be harmful to the health of a significant proportion of our patients.

\section{ORIGINS}

The BMI can be traced back, rather surprisingly, to a famous Belgian. Adolphe Quetelet's interest in the emerging discipline of statistics in the mid 1830s saw him collect data on men's heights and weights at various ages. From this study, which he hoped would allow him to determine the 'average' man, he formulated what became known as the Quetelet formula, but which is now known as the BMI, and is calculated by dividing an individual's weight in kilograms by their height in meters squared. It can all seem so clear and precise that it may be something of a surprise to discover that its proper use in contemporary clinical practice generally requires that other factors also be taken into account. However, these factors are invariably ignored. In short, it was designed with Belgians in mind, and does not work so straightforwardly on such populations as Bangladeshis, Botswanans, or boxers. If its use is to properly help determine whether someone is obese and hence in need of some intervention to help restore health, or to reduce their risk of ill health, then those who use it should understand it rather better than most of them seem to do.

\section{'FATTIES'}

The BMl is a surrogate measure of fatness, and as fatness is an indicator of health ('if you have a BMI of over 25 , you have an increased risk of serious health problems'1), it is presumably to the essence of its use that the BMI can help identify 'fatties' in a potentially nonthreatening, almost politically-correct way. Its appearance of scientific impartiality allows it to help maintain a patient-clinician relationship where a statement based on simple clinical observation alone may come across as indelicate.

The generally accepted view is that a BMI of 25-29.9 indicates that one is 'overweight', while 30 or above classifies one as obese; having a BMl above 35 suggests one is 'grossly obese', so that even for routine elective surgery, such a patient may need to discuss their options with the anaesthetist. BMI above 40 is 'bariatric' or 'morbidly obese' and may well need hoists to help mobilise. At the other end of the scale, anything below 18.5 is also generally regarded as distinctly unhealthy: a score below 17.5 in fact might well indicate anorexia, and 15 is usually taken as the upper limit of 'starvation'. As for a point of reference, a BMI of 18 became, after much debate and controversy, the minimum acceptable measurement for models in the 2006 Milan fashion week.

\section{'BIG BONES'}

But numbers, and statistics, can sometimes mislead, and in fact there are occasions when the human eye can be better at adjudicating obesity than the BMI. Take a professional boxer such as Frank Bruno. He may technically have a high BMI but that will comprise a lot of lean muscle and, accordingly, this should count as physically healthy. In the other corner though, Ricky Hatton's BMI apparently fluctuates fantastically as he weaves in and out of training and excesses of curry and lager, such that one day his high BMI might be due to a good muscle-to-fat ratio while a few months, after the last big fight, a similar BMI might hide a very different bodily composition. The point clearly is that a 'high' BMI does not always indicate obesity, and we should expect clinicians to use their common sense.
Certainly, increased amounts of fat (especially around the abdomen) increase the dangers of unhealthiness. However, $\mathrm{BMI}$ fails to distinguish fat adequately from fat-free mass, such as muscle and bone and other bodily tissue. In fact, a higher $\mathrm{BMI}$ really could be due to having 'big bones' after all!

I do not know why the Quality and Outcomes Framework has not yet made more of the obesity register than it has. One could, though, speculate that it is because someone in the Department of Health has realised that it is not a simple matter of 'BMl>29.9 = obesity', as racial differences, for instance, are also significant:

'Many Asian races, particularly those from the Indian subcontinent, tend to carry a proportionately higher fat mass for a given BMI than Caucasians. The reverse is true for most black people and for Polynesians. ${ }^{2}$

Thus, a BMI of 23 can be 'unhealthy' for Asians who can also be regarded as obese with a BMI of just 25 .

Age is also an important factor. It is well recognised that the $\mathrm{BMI}$ is not valid for those who have yet to attain their twentieth year. Under- and overweight children are categorised by reference to percentile charts, with those in the bottom $4 \%$ usually being regarded as underweight, while those in the top $15 \%$ are likely to be considered as overweight; consideration will be given to the physical dimensions of the parents. The older people get the greater the likelihood too that their bones may begin to become osteoporotic and so less dense. Yet, no adjustment of the BMI calculation of weight in kilogrammes divided by height in meters squared is thought necessary to accommodate this fact. However, it is the case that while someone ages their BMl might remain stable (lets assume their height does too), the percentage of fat they contain is likely 
to increase, and this can become significant above the age of 60 years. And let us be clear:

'Fatness is a risk factor for ischemic heart disease, congestive heart failure, stroke, cancer, respiratory disease, diabetes, hyperlipidemia, hypertension, asthma, sleep apnea, arthritis, degenerative joint disease, gastric reflux, and depression."

Presumably it is because of this tendency of fatness to increase the risk of morbidity that there is an 'obesity' category in QOF. It is well known for instance, that type 2 diabetes is closely linked to obesity such that everyone who is overweight has a high risk for developing the condition. It is also now widely accepted that both obesity and type 2 diabetes are particularly prevalent amongst Asians living in the West. Part of this problem is that they become obese more readily than other ethnic groups (as we have seen, they become obese and overweight at lower BMI levels than others). Yet, by delaying intervention until their BMI has attained Western levels (that is, a BMI of 30 or more) they are well on their way to developing such morbidities as excess adiposity can dispose one. By using $\mathrm{BMI}$ in a non-ethnically sensitive way, we are failing these patients.

\section{A SIMPLE SOLUTION}

If the objective of the obesity register within QOF is to help to tactfully identify the unhealthy (those with more body fat than is good for them) then rather than using the BMI score, there is another simple tool which could be used to better help establish this and that is the percentage body fat method. The bioimpedence analysers used for this offer a significant, and practical improvement on the BMI, and are readily available from such stores as stock bathroom scales.
'Bioimpedence is probably the only technique that can meet the criteria of being simple, rapid and free from operator variability. The foot-to-foot technique is especially simple ... and provides more accurate assessments of body fat than predictive equations based on BMI.' ${ }^{2}$

With this method there are widely recommended cut-offs for obesity at $25 \%$ fat for men and $30 \%$ for women and at the other end of the scale $5 \%$ fat for men and $12 \%$ for women. ${ }^{4}$ Defining obesity as having a body fat range of between $5-25 \%$ (male) and $12-30 \%$ (female) is very simple and works with any race and adult age.

While NICE guidelines recognise that:

... the concept of different [BMI] cutoffs for different ethnic groups has been proposed by the WHO, ... [because] there is ongoing debate. ${ }^{5}$

NICE have felt unable to give tailored guidance. However, this is not good enough. There may be uncertainty, but no harm and only good can come from using more ethnic-specific BMI levels with those groups most at risk. Indeed, the ethical principle of justice, as for instance formulated by Aristotle's principle of equality, ${ }^{6}$ requires that not only should equals be treated equally, but also unequals should be treated unequally in relevant respects and in proportion to the relevant inequalities that afflict them.

Clearly, by using a Euro-centric determinant of obesity, many of those who might most benefit - in particular those Asians who should be regarded as obese at a lower cut-off - lose most. This fact has received too little attention to date.

No one really benefits from an increasingly unhealthy society and general practice can certainly do more to help address the problem of obesity and the diseases it can cause among those ethnic groups most at risk. A first step could be to rethink the use of the $\mathrm{BMI}$ among the Asian community.

\section{Stephen Humphreys}

\section{REFERENCES}

1. Stuttaford T. The bigger picture. The Times 2008; 17 April (times2): 6 .

2. Prentice AM, Jebb SA. Beyond body mass index. Obesity Reviews 2001; 2: 141-147.

3. Buckhauser RV, Cawley J. Beyond BMI: the value of more accurate measures of fatness and obesity in social science research. J Health Econ 2008; 27: 519-529.

4. Digate Muth N. What are the guidelines for percentage of body fat loss? America's Authority on Fitness 2009, 2 Dec: http://www.acefitness.org/blog/112/what-are-theguidelines-for-percentage-of-body-fat/ (accessed 3 Aug 2010).

5. National Institute for Health and Clinical Excellence. Obesity: guidance on the prevention, identification, assessment and management of overweight and obesity in adults and children. CG043.

http://www.nice.org.uk/nicemedia/live/11000/30361/30 361.doc (accessed 27 Jul 2010).

6. Aristotle. The Nicomachean Ethics. London: Penguin Classics, 2004.

DOI: 10.3399/bjgp10X515548 